\title{
Campylobacter Y CAMPYLOBACTERIOSIS: UNA MIRADA DESDE AMÉRICA DEL SUR
}

\author{
Heriberto Fernández ${ }^{1, a}$
}

\begin{abstract}
RESUMEN
Las especies termotolerantes de Campylobacter han adquirido gran importancia en la salud pública, por ser considerados agentes de diarrea infecciosa para el ser humano. En esta breve revisión se presenta información sobre aspectos epidemiológicos, clínicos y bacteriológicos de campylobacteriosis en América del Sur. Asimismo, se señalan algunas diferencias con relación a su presentación en países industrializados.
\end{abstract}

Palabras clave: Campylobacter, Infecciones por Campylobacter, Diarrea, Epidemiología, Zoonosis (fuente: DeCS BIREME)

\section{Campylobacter AND CAMPYLOBACTERIOSIS: A VIEW FROM SOUTH AMERICA}

\begin{abstract}
The thermotolerant species of Campylobacter have become very important in public health, especially as agents of infectious diarrhea in human beings. In this brief revision we present part of the available information generated in South America about epidemiological, clinical and bacteriological aspects of campylobacteriosis and we identify some differences between the observed and documented campylobacteriosis in South America compared to those described in industrialized countries.
\end{abstract}

Key words: Campylobacter, Campylobacter Infections, Diarrhea, Epidemiology, Zoonoses (source: MeSH NLM)

En las últimas décadas, las especies termotolerantes de Campylobacter (C. jejuni, C. coli, C. lari y más recientemente $C$. upsaliensis) han adquirido gran importancia en salud pública, particularmente como agentes de diarrea infecciosa para el ser humano ${ }^{(1)}$.

En esta breve revisión, presentaremos parte de la información que, sobre aspectos epidemiológicos, clínicos y bacteriológicos de Campylobacter ha sido generada desde América del Sur. Con ello pretendemos incentivar en nuestra región la investigación sobre Campylobacter, reconocer el trabajo realizado por científicos de la región y señalar algunas diferencias en las características de la campylobacteriosis observadas y documentadas en América del Sur con respecto a aquellas descritas en países industrializados.

El Campylobacter jejuni es la especie más frecuentemente aislada, tanto en los países en vías de desarrollo como en los países industrializados y, en estos últimos, C. coli es reconocido como agente de diarrea entre el 5 y el $10 \%$ de todos los casos de diarrea por Campylobac- ter. Sin embargo, en Sudamérica, Campylobacter coli ha sido aislado con mayor frecuencia, representando cerca del $25 \%$ de los casos de diarrea producidos por especies del género (Tabla 1). En vista que en la región, $C$. coli ha sido aislado a partir de agua de río, de hígados de pollo para consumo humano y de diversos reservorios animales -tanto domésticos como silvestres, incluyendo especies de la fauna amazónica-, materia fecal de pollos, carne de aves y aguas servidas (Tabla 2), es posible que exista una vinculación del medio ambiente y el consumo de alimentos con la mayor frecuencia de aislamiento de C. coli como agente de diarrea en esta región.

Otra diferencia es el hallazgo frecuente de portadores sanos en países sudamericanos. En contraposición, en países industrializados, el estado de portador sano es raro y de muy baja presentación (Tabla 1).

Probablemente, el estado de portador sano de Campylobacter en países de América del Sur se relaciona con aspectos ligados a bajas condiciones de saneamiento básico, las que pueden promover

\footnotetext{
1 Instituto de Microbiología Clínica, Facultad de Medicina, Universidad Austral de Chile, Valdivia, Chile.

a Tecnólogo Médico, Doctor en Ciencias, mención Microbiología.
} 
Tabla 1. Frecuencia de aislamiento (\%) de C. jejuni y C. coli en niños con diarrea y en niños normales (controles) en países de América del Sur.

\begin{tabular}{|c|c|c|c|c|c|}
\hline \multirow{2}{*}{ País } & \multicolumn{2}{|c|}{ C. jejuni } & \multicolumn{2}{|c|}{ C. coli } & \multirow{2}{*}{ Referencia } \\
\hline & Diarrea & Control & Diarrea & Control & \\
\hline Argentina & 4,6 & NE & $1,4[30,4]$ & NE & Notario et al. (59) \\
\hline Argentina & 9,1 & NE & $\mathrm{NAI}$ & NE & López et al. (54) \\
\hline Argentina & 30,1 & NE & $\mathrm{NAI}$ & NE & Giugno y Oderiz (60) \\
\hline Bolivia & 10,5 & 9,6 & NAI & NE & López y Valentina (61) \\
\hline Bolivia & 4,4 & NE & $7,3[45,4]$ & NE & WHO (1) \\
\hline Brasil & 5,8 & 4,9 & $2,2[37,9]$ & 2,0 & Fernández et al. (62) \\
\hline Brasil & 9,6 & 7,2 & $6,0[38,5]$ & 1,2 & da Silva Quetz et al. (63) \\
\hline Chile & 9,2 & 4,0 & NAI & NE & Fernández (64) \\
\hline Chile & 5,7 & NE & $\mathrm{NAI}$ & NE & Figueroa et al. (65) \\
\hline Chile & 14,1 & 4,0 & $5,4[27,7]$ & 3,6 & WHO (1) \\
\hline Colombia & 14,4 & 3,7 & $2,4[14,3]$ & 1,2 & Ordóñez (66) \\
\hline Colombia & 2,3 & NE & NAI & NE & Manrique et al. (67) \\
\hline Ecuador & $23,0^{*}$ & NE & NAI & NE & Guderian et al. (68) \\
\hline Paraguay & 18,4 & NE & 0,6 & NE & Weiler et al. (69) \\
\hline Perú & $15-23$ & $\mathrm{NE}$ & NAI & NE & Grados et al. (2) \\
\hline Perú & 18,2 & $13,813,8$ & NAl & NE & Murga et al (5) \\
\hline Perú & $13^{*}$ & NE & $\mathrm{NAI}$ & NE & Perales et al. (70) \\
\hline Perú & 2,9 & NE & $5,0[63,3]$ & NE & Castillo et al. (71) \\
\hline Uruguay & 14,3 & NE & NAI & NE & Mota et al. (72) \\
\hline Venezuela & $13,0^{*}$ & $9^{*}$ & NAI & NE & Urrestarazu et al. (73) \\
\hline Venezuela & 6,5 & & $\mathrm{NAI}$ & $\mathrm{NE}$ & Díaz et al. (74) \\
\hline
\end{tabular}

* Referido como C. jejunilcoli o Campylobacter spp.

NE No estudiado

NAI No aislado o identificado

[ ] Frecuencia de C. coli en relación a los casos de diarrea por Campylobacter

mayores oportunidades de transmisión de las especies diarrogénicas de Campylobacter, especialmente a niños de corta edad, desde sus reservorios y fuentes de contaminación ${ }^{(2-4)}$.

En general, los síntomas y signos clínicos observados en los pacientes de países industrializados y en aquellos de países en desarrollo, no difieren. Sin embargo, en los países en desarrollo es posible encontrar una alta frecuencia de deshidratación, situación clínica que probablemente pueda estar asociada con el bajo estado nutricional diagnosticado en la mayoría de los pacientes incluidos en los ensayos clínicos ${ }^{(5-9)}$.

Esta información comienza a ser estructurada a partir de los primeros aislamientos de Campylobacter en niños con diarrea, realizados por Ricciardi en Brasil el año $1979{ }^{(10)}$. Posteriormente, estas bacterias han sido aisladas como agentes enteropatógenos en prácticamente todos los países del continente sudamericano, en los cuales Campylobacter aparece como causa importante de cuadros diarreicos, predominantemente en niños menores de dos años, disminuyendo su frecuencia a medida que los niños aumentan en edad. Concomitantemente, van apareciendo portadores asintomáticos, en frecuencias variables (Tabla 1 ).
Con respecto a métodos diagnósticos, muchos investigadores sudamericanos se han interesado en la evaluación e introducción de técnicas y procedimientos de laboratorio, simples y asequibles, para promover la investigación y el diagnóstico de Campylobacter en laboratorios con limitación de recursos. En Brasil, Magalhães et al., propusieron un método sencillo para la obtención de la atmósfera apropiada para el cultivo de Campylobacter ${ }^{(11)}$. Fernández y Trabulsi compararon el rendimiento de dos medios de cultivo incubados bajos diferentes condiciones atmosféricas ${ }^{(12)}$. Por su parte, investigadores de Argentina y Chile ${ }^{(13)}$ han propuesto un nuevo medio de cultivo adicionado de lisado globular mientras que científicos del Perú (14) han evaluado el uso alternativo de sangre de cerdo y de bovino para el aislamiento de Campylobacter.

El uso de métodos directos para el diagnóstico presuntivo y la eficiencia de dos tipos de toma de muestras fecales también fueron evaluados en América del Sur. El método de Gram directo ${ }^{(15)}$, la microscopía de contraste de fase ${ }^{(12)}$ y las tinciones de Hooker (16) y de Vagó ${ }^{(17)}$ demostraron ser útiles para el diagnóstico presuntivo en la diarrea por Campylobacter, encontrándose un valor predictivo positivo de 60 y $85,7 \%$ en las dos primeras y una sensibilidad del $37 \%$ y especificidad del $100 \%$ en 
Tabla 2. Frecuencia de aislamiento (\%) de C. jejuni y $C$. coli de animales, alimentos, aguas superficiales y aguas servidas en algunos países de América del Sur.

\begin{tabular}{|c|c|c|c|c|}
\hline País & $\begin{array}{l}\text { C. jejuni / } \\
C \text { coli sp }\end{array}$ & C. jejun & C. coli & Referencias \\
\hline $\begin{array}{l}\text { Argentina } \\
\text { bovinos } \\
\text { pollos } \\
\text { cerdos } \\
\text { perros } \\
\text { gatos } \\
\text { carne de pollo } \\
\text { carne bovina }\end{array}$ & $\begin{array}{c}1,7 \\
5,9 \\
16,96 \\
20,0 \\
3,2\end{array}$ & 94,0 & 16,6 & $\begin{array}{c}\text { Piazza et al. (75) } \\
\text { Giacoboni et al. (76) } \\
\text { (75) } \\
\text { López et al. (54) } \\
(76) \\
(76) \\
(75)\end{array}$ \\
\hline $\begin{array}{l}\text { Brasil } \\
\text { perros } \\
\text { gatos } \\
\text { bovinos } \\
\text { pollos } \\
\text { carne de pollo } \\
\text { carne de cerdo } \\
\text { monos } \\
\text { aguas servidas }\end{array}$ & 35,0 & $\begin{array}{r}35,2 \\
23,8 \\
53,3 \\
66,9 \\
68,8 \\
\\
7,6 \\
56,2\end{array}$ & $\begin{array}{c}7,6 \\
7,9 \\
8,4 \\
19,8 \\
8,3 \\
\\
6,1 \\
25,0\end{array}$ & $\begin{array}{c}\text { Fernández (77) } \\
(77) \\
\text { Modolo et al (78) } \\
\quad(77) \\
\text { Kuana et al. (79) } \\
\text { Almeida et al. (80) } \\
\text { Andrade et al. (81) } \\
\text { Filgueiras et al. (82) }\end{array}$ \\
\hline $\begin{array}{l}\text { Chile } \\
\text { perros } \\
\text { bovinos } \\
\text { cerdos } \\
\text { pollos } \\
\text { patos } \\
\text { gansos } \\
\text { gorriones } \\
\text { aves acuáticas } \\
\text { hígados de pollo } \\
\text { carne de pollo } \\
\text { agua de río }\end{array}$ & $54,0-90,0$ & $\begin{array}{l}34,7 \\
22,5 \\
15,0 \\
45,0 \\
66,0 \\
28,4 \\
28,0 \\
11,8 \\
21,7 \\
\\
9,5\end{array}$ & $\begin{array}{c}16,7 \\
7,5 \\
55,0 \\
15,0 \\
7,0 \\
18,3 \\
12,0 \\
11,8 \\
69,6 \\
24,3\end{array}$ & 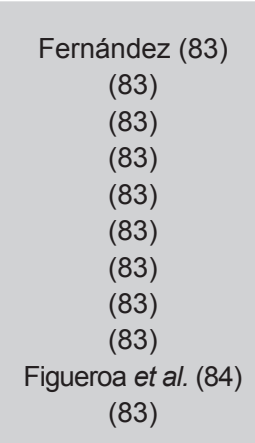 \\
\hline $\begin{array}{l}\text { Colombia } \\
\text { leche }\end{array}$ & & 4,0 & 1,0 & Ordóñez (66) \\
\hline $\begin{array}{l}\text { Perú } \\
\text { perros } \\
\text { gatos } \\
\text { patos } \\
\text { pollos } \\
\text { monos (mascotas) } \\
\text { monos silvestres } \\
\text { loros } \\
\text { fauna amazónica }\end{array}$ & & $\begin{array}{c}25,0 \\
21,0 \\
18,2 \\
61,4 \\
10,6 \\
4,6 \\
4,9 \\
5,5\end{array}$ & $\begin{array}{c}21,3 \\
16,3 \\
\\
2,1 \\
5,5\end{array}$ & $\begin{array}{l}\text { Grados et al. (2) } \\
(2) \\
(2) \\
(2) \\
\text { Tresierra et al. (85) } \\
\text { (85) } \\
\text { Tresierra et al. (86) } \\
\text { Tresierra et al. (87) }\end{array}$ \\
\hline
\end{tabular}

la de Hooker, mientras que con la tinción de Vagó fue posible el diagnóstico presuntivo de Campylobacter en el $3,63 \%$ de las muestras estudiadas. Por otra parte, un estudio realizado en Brasil, demostró que la toma de muestra mediante hisopado rectal tenía la misma eficiencia en el aislamiento de Campylobacter que la muestra obtenida por emisión fecal espontánea ${ }^{(18)}$.
En América del Sur han sido utilizados prácticamente todos los medios de cultivo selectivos descritos en la literatura y, adicionalmente, se ha utilizado el método de filtración pasiva sobre agar sangre, lo que ha permitido el aislamiento, en muestras fecales de animales (domésticos y silvestres) y de seres humanos, de las especies tradicionales de Campylobacter y de otras especies menos comunes como C. upsaliensis, C. fetus, C. jejuni subsp. doylei y C. insulaenigrae ${ }^{(17,19-26)}$.

Con respecto a los mecanismos de patogenicidad, tanto la adherencia como la invasión y la producción de substancias tóxicas en cepas de Campylobacteraisladas de seres humanos, animales y otras fuentes, han sido demostradas en diferentes países sudamericanos, utilizando cultivos celulares o modelos animales ${ }^{(27-36)}$.

El comportamiento de Campylobacter frente a factores externos también ha sido preocupación de investigadores sudamericanos, existiendo datos sobre la resistencia a la desecación y a otras situaciones ambientales ${ }^{(37,38)}$ y su sobrevida en suero humano ${ }^{(39)}$, en leche ${ }^{(40,41)}$ y en medios de transporte sometidos a diferentes condiciones ambientales ${ }^{(42)}$

El estudio del comportamiento de Campylobacter a diferentes antimicrobianos ha sido motivo de interés de investigadores de varios países sudamericanos, quienes han puesto en evidencia la aparición de cepas de origen humano y animal, resistentes a eritromicina, tetraciclina, ampicilina y a quinolonas, encontrándose que la resistencia a estas últimas ha sido explosiva, alcanzando tasas superiores al $60 \%$ (Tabla 3$)^{(1,43-53)}$.

El papel de las especies enteropatogénicas de Campylobacter en la etiología de la enfermedad entérica humana ha sido bien establecido tanto en los países industrializados como en los que se encuentran en desarrollo. Sin embargo, en estos últimos todavía no se conocen bien sus vías de transmisión, existiendo consenso que el medio ambiente y el nivel de saneamiento básico tienen importancia en la transmisión de estos agentes ${ }^{(1)}$. Los trabajos de Grados et al. en Perú $(2,3)$

Tabla 3. Resistencia de Campylobacter (\%) a algunos antimicrobianos en cinco países de América del Sur $(1,43-53,88,89)$

\begin{tabular}{lcccc}
\hline \multicolumn{1}{c}{ País } & Ampicilina & Eritromicina & Tetraciclina & Quinolonas \\
\hline Argentina & 47,2 & 6,3 & 40,8 & $49,1-59,6$ \\
Bolivia & $\mathrm{NI}$ & 61,4 & 65,9 & 47 \\
Brasil & $18-26,9$ & $9,1-38,9$ & $9,1-43$ & $14-72,2$ \\
Chile & $4,6-25$ & $5,6-58,6$ & $1,8-15,1$ & $5,3-50$ \\
Perú & 37 & 17 & $\mathrm{NI}$ & $63-78$ \\
\hline
\end{tabular}

NI: no informado 
fueron pioneros para Sudamérica en la identificación de factores de riesgo y vehículos de transmisión de estas bacterias, asociando factores ambientales con la prevalencia de la infección. Posteriormente, los trabajos de Oberhelman et al. (4) y Murga et al. (5), también en Perú y López et al. ${ }^{(54)}$ en Argentina, aportan mayores antecedentes a este respecto, al igual que Araya et al. (55) en Chile, quienes establecen que la infección por Campylobacter es más frecuente en preescolares de estrato socioeconómico bajo que en aquellos de estratos altos que viven en áreas de buenas condiciones de saneamiento básico. Un fenómeno similar fue demostrado en animales (gallinas, perros y bovinos) por Fernández et al. (56-58) aislando estas bacterias con frecuencias significativamente mayores en aquellos animales que vivían o eran mantenidos en condiciones de bajo saneamiento básico.

De esta revisión de la literatura biomédica sudamericana se concluye que varias especies de Campylobacter - especialmente las especies termotolerantes - son agentes importantes de diarrea en esta región, las que están ampliamente diseminados en la naturaleza, con una extensa gama de reservorios animales, domésticos y silvestres, constituyendo un problema de salud pública que aún no ha sido bien dimensionado por las instancias sanitarias pertinentes, a pesar de la vasta y valiosa información generada en los países sudamericanos sobre aspectos bacteriológicos, patogénicos, clínicos y epidemiológicos de la campylobacteriosis.

El intercambio de información entre científicos de Sudamérica sobre los diferentes aspectos de la campylobacteriosis y la ejecución de estudios colaborativos son elementos de la mayor importancia para entender mejor el complejo problema que representan, para los países sudamericanos, las especies de Campylobacter y sus infecciones asociadas y, a la vez, constituirían una contribución para las autoridades sanitarias a la hora de diseñar y enfocar las estrategias de prevención, control y manejo del riesgo epidemiológico de la campylobacteriosis.

\section{Conflictos de Interés}

El autor declara no tener conflictos de interés en la publicación del presente artículo.

\section{REFERENCIAS BIBLIOGRÁFICAS}

1. World Health Organization. The increasing incidence of human Campylobacteriosis. Report and Proceedings of a WHO Consultation of Experts. Copenhagen. WHO/CDS/ CSR/APH/2000.4; 2001. p.

2. Grados O, Bravo N, Black RE, Butzler JP. Paediatric Campylobacter diarrhoea from household exposure to live chickens in Lima, Peru. Bull WHO. 1988; 66 (3): 369-74.
3. Grados O, Bravo N, Black RE, Butzler JP. Diarrea pediátrica por Campylobacter debida a la exposición doméstica a pollos vivos en Lima, Perú. Bol Of Sanit Panam. 1989; 106 (3): 205-13.

4. Oberhelman RA, Gilman RH, Sheen P, Cordova J, Taylor DN, Zimic M, et al. Campylobacter Transmission in a Peruvian Shantytown: A Longitudinal Study Using Strain Typing of Campylobacter Isolates from Chickens and Humans in Household Clusters. J Infect Dis. 2003; 187(2): 260-9.

5. Murga H, Huicho L, Guevara G. Acute Diarrhoea and Campylobacter in Peruvian Children: a Clinical and Epidemiologic Approach. J Trop Ped. 1993; 39 (6): 338-41.

6. Cordero P, Araya M, Espinoza J, Figueroa G, Pacheco I, Brunser O. Efecto de la hidratación oral y realimentación precoz en la evolución de la diarrea aguda del Lactante. Rev. Chil. Pediatr. 1985; 56 (6): 411-7.

7. Murga H. Diarrea aguda y Campylobacter fetus. Aspectos clínicos en 78 niños del Instituto de Salud del Niño [Tesis Doctoral]. Lima: Facultad de Medicina, Universidad Nacional Mayor de San Marcos; 1988.

8. Figueroa G, Araya M. Symptomatic and asymptomatic infections due to Campylobacter jejuni. Rev Chil Pediatr. 1985; 56(6): 486-490.

9. Soza G, Ossa G, Illesca V, Reydet P, Inostroza J, Rodriguez J. Infantile acute diarrhea due to Campylobacter jejuni. Rev Méd Chile. 1987; 115(1): 19-23.

10. Ricciardi ID, Ferreira MCS, Otto SS, Oliveira N, Sabra A, Fontes CF. Thermophilic Campylobacter-associated diarrhoea in Rio de Janeiro. Rev Bras Pesq Méd Biol. 1979; 12(2-3): 189-91.

11. Magalhães M, Andrade MA, Silva GP. Simple and inexpensive method for culturing Campylobacter fetus subsp jejuni. Rev Microbiol. 1982; 13(2): 124-5.

12. Fernández $\mathbf{H}$, Trabulsi LR. Comparison of two culture media and value of direct microscopic examination for the diagnosis of the intestinal infection due to Campylobacter jejuni/coli. Rev Microbiol. 1990; 21: 49-53.

13. Fernández H, Notario R, Gambande T, Borda N, Rivera S. Evaluation of a new medium with globular lysate for the isolation of Campylobacter jejuni and Campylobacter coli. Rev Arg Microbiol. 1990; 22(2): 73-79.

14. Tresierra-Ayala A, Navas M, Flores J, Perea R, Huanaquiri J, Bendayán M, et al. Growth capacity of thermotolerant campylobacters in culture media supplemented with pig and cow blood. Braz Arch Biol Technol. 2010; 53(5): 1087-91.

15. Pavan MFV, Mamizuka EM. Rapid (presumptive) diagnosis of acute diarrhea caused by thermophilic Campylobacter $\mathrm{sp}$. Rev Farm Bioquim Univ Sáo Paulo. 1987; 23(2): 152-158

16. Chanqueo L, García P, León E, Blu A. Evaluación de la tinción de Hucker para la búsqueda rutinaria de Campylobacter sp en el estudio de un síndrome diarreico agudo. Rev Chil Infect. 2005; 22 (3): 242-246.

17. Patiño L. Estudio comparativo entre la microscopía convencional y el método de cultivo con microfiltros para la identificación del Campylobacter sp. en muestras fecales de pacientes del HNGAI durante el $2^{\circ}$ semestre del 2002. [Tesis especialista en patología clínica]. Lima: Facultad de Medicina, Universidad Nacional Mayor de San Marcos; 2002.

18. Canduro PF, Mezzari A, Dias CAG. Stool culture: prevalence of pathogenic microorganisms in a private laboratory in Porto Alegre, Brazil. Rev Bras Patol Clin. 1987; 23:152-6. 
19. Modolo JR, Fernandes Margato LF, Gottschalk AF, Magalhães Lopes CA. Incidence of Campylobacter in pigs with and without diarrhea. Rev Microbiol. 1999; 30(1):19-21.

20. Fernández $\mathbf{H}$, Rodríguez $\mathbf{R}$, Barudi $\mathbf{C}$, Lobos $\mathbf{M}$. A case of acute diarrhea due to the emerging pathogen Campylobacter jejuni subsp. jejuni in Southern Chile. Braz J Microbiol. 2003; 34: 52-54.

21. Modolo JR, Giuffrida R. Campylobacter upsaliensis isolated from young dogs with and without diarrea. Rev Soc Bras Med Trop. 2004; 37(1):72-73.

22. Giuffrida R, Modolo JR, Araújo Junior JP, Duarte JMB. Campylobacter sp. in feces of marsh deer (Blastocerus dichotomus). Arq Inst Biol São Paulo, 2004; 71:7-8.

23. Fernández H, Vera F, Villanueva MP. Especies de Arcobacter y Campylobacter en aves y mamíferos del sur de Chile. Arch Med Vet. 2007;39(2):163-165

24. Fernández H, Vera F, Villanueva MP, García A. Occurrence of Campylobacter species in healthy well-nourished and malnourished children. Braz J Microbiol. 2008;39:56-8.

25. González M, Villanueva MP, Debruyne L, Vandamme $\mathbf{P}$, Fernández H. Campylobacter insulaenigrae: first isolation report from South American Sea Lion (Otaria flavescens, (Shaw, 1800). Braz J Microbiol. 2011; 42: 261-5

26. Díaz C, Vizcaya L, Velasco J. Evaluación de un medio y la técnica de filtración para el aislamiento de Campylobacter sp. en un grupo de riesgo. Rev Fac Farm. 2003; 45 (1): 14-18.

27. Fernández H, Trabulsi L. Invasive and enterotoxic properties in Campylobacter jejuni and Campylobacter coli strains isolated from humans and animals. Biol Res. 1995; 28(3):205-10

28. Andrews E, Fernández H, Folch H, Eller G, Pollette M. Evidencias de la participación de epítopes flagelares de Campylobacter jejuni en la adhesión celular in vitro. Medicina (Bs. As.) 1996; 56(5):487-92.

29. Fernández H,Eller G, Freymuller E, Vivanco T. Detection of Campylobacter jejuni invasion of HEp-2 cells by acridine orange-crystal violet staining. Mem Inst Oswaldo Cruz. 1997; 92(4): 509-511.

30. Velasco J, Vizcaya L, Blanco N, Pérez I. Adherencia e invasión de campilobacterias termotolerantes en células HEp-2. Rev Soc Venez Microbiol. 2002; 22 (1):32-36.

31. Fernández $\mathbf{H}$, Vivanco $\mathbf{T}$, Eller $\mathbf{G}$. Expression of invasiveness of Campylobacter jejuni ssp. jejuni after serial intraperitoneal passages in mice. J Vet Med B. 2000; 47(8):635-639.

32. Maggi L, Martínez J, Prado V, González L, Riveros V Prevalence of Campylobacter jejuni/coli in caretakers and animals from the Santiago Zoo. Rev Méd Chile. 1988; 116(1): 7-12.

33. FernándezH,ToroJ.Enterotoxigenicidad deCampylobacter jejuni subsp. jejuni y su relación con aumento de AMPc y alteración electrolítica en el intestino de ratas. Rev Méd Chile. 1998; 126(8): 919-23.

34. Tresierra-Ayala A, Fernández H, Zamora J. Enterotoxigenic capacity of Campylobacter coli strains isolated from humans and bovines. Mem Inst Oswaldo Cruz. 1996; 91(5): 619-620.

35. Darc da Silva Thomé J. Citotoxinas e hemolisinas produzidas por Campylobacter jejuni isolados de diferentes origens. [Tesis de Maestría] Campinas: Mestrado em Genética e Biologia Molecular. Universidade Estadual de Campinas; 2006
36. De Carvalho AF. Detecção dos genes da toxina citoletal distensiva (CDT) em estirpes de Campylobacter jejuni subsp. jejuni isoladas de frangos de corte e hortaliças. [Tesis de Maestría] São Paulo: Programa de Pós-Graduação Sanidade Animal, Segurança Alimentar e o Ambiente. Instituto Biológico; 2009.

37. Fernández $\mathbf{H}$, Vergara M, Tapia F. Dessication resistance in thermotolerant Campylobacter species. Infection. 1985; 13(4):197.

38. Tresierra A, Fernández H, Bendayán ME, Bernuy A, Espinoza F. Influencia de las condiciones ambientales sobre la frecuencia de infección por especies termotolerantes de Campylobacter en pollos. Rebiol. 1995; 15: 39-43,

39. Fernández $\mathbf{H}$, Giusti $\mathbf{G}$, Bertoglio JC. Effect of the complement system on the sensitivity of Campylobacter jejuni and Campylobacter coli to human blood serum. Braz J Med Biol Res. 1995; 28(2):227-9.

40. Tresierra-Ayala A, Ruiz R, Bendayan M, Fernández H. Survival times of Campylobacter coli in sterilized buffalo milk. J Vet Med B. 1999;46:141-4.

41. Tresierra-Ayala $A$, Guzmán $A$, Fernández H. survival rate of Campylobacter coli strains in sterile buffalo and bovine milk. Arch Med Vet. 2001; 33(2): 111-4.

42. Tresierra-Ayala A, Grandez C, Bendayan ME, Fernández H. Survival rates of thermotolerant Campylobacter species in a transport and enrichment medium under different environmental conditions. Arq Bras Med Vet Zootec. 2006;58: 662-4.

43. Figueroa G, Troncoso M, Galeno H, Soto V, Toledo MS. Biotypes, serogroups and antibiotic susceptibility of Campylobacter jejuni and Campylobacter coli in Chile. J. Infect. 1990; 20(2):123-7.

44. Fernández H, Mansilla M, González V. Antimicrobial susceptibility of Campylobacter jejuni subsp. jejuni assesed by E-test and double dilution agar method in Southern Chile. Mem Inst Oswaldo Cruz. 2000;95(2):247-9.

45. Giacoboni GI, Cerdá R, López C. Emergencia a la resistencia antibiótica en cepas de Campylobacter jejuni aisladas de carne de pollo. Analecta Veterinaria. 2001;21(1): 63-7.

46. Aquino MHC, Filgueiras, ALL, Ferreira MCS, Oliveira SSD, Bastos MC, et al. Antimicrobial resistence and plasmid profiles of Campylobacter jejuni and Campylobacter coli from human and animal sources. Lett Appl Microbiol. 2002;34 (2):149-53.

47. Kelley CP. Estrategias prácticas para la vigilancia epidemiológica. En: Universidad de South Florida. La emergencia de patógenos resistentes: Un llamado a la colaboración interventiva. PharmaCom Group, Inc; 2002. p.7-12.

48. Modolo JR, Giuffrida R, Lopes C. Antimicrobial susceptibility of 51 Campylobacter strains isolated fron diarrheic and diarrhea-free dogs. Arq Inst Biol São Paulo. 2003; 70 (3): 283-6.

49. Rivera N, Fernández H, Bustos R, Valenzuela ME, Trabal $\mathbf{N}$, Montenegro S, et al. Susceptibilidad antimicrobiana de cepas de Campylobacter aisladas de carcasas de aves, sangre y fecas humanas. Rev Latinoam Actual Biomédi. 2007;1(2):32-7.

50. Miranda KL, Lage AP. Antimicrobial susceptibility of Campylobacter sp strains isolated from calves with and without diarrhea in Minas Gerais state, Brazil. Braz J Microbiol. 2007;38(2):357-362. 
51. Kuana SL, dos Santos LR, Rodrigues LB, Borsoi A, do Souza Moraes HL, Salle CT, et al. Antimicrobial resistance in Campylobacter spp isolated from broiler flocks. Braz J Microbiol. 2008; 39(4): 738-40.

52. Lopes GV. Campylobacter spp. no abate e no varejo: ocorrência em carcaças de bovinos para exportação e em corte refrigerados de aves e bovinos. [Tesis de Maestría] São Paulo: Faculdade de Ciências Farmacêuticas. Univeridade de São Paulo; 2009.

53. Pantozzi FL, Moredo FA, Vigo GB, Giacoboni GI. Resistencia a los antimicrobianos en bacterias indicadoras y zoonóticas aisladas de animales domésticos en Argentina. Rev Argent Microbiol. 2010; 42(1): 49-52.

54. López C, Agostini A, Giacoboni G, Cornero F, Tellechea D, Trinidad JJ. Campilobacteriosis en una comunidad de bajos recursos de Buenos Aires, Argentina. Rev Sci Tech Off Int Epiz. 2003; 22 (3):1013-20.

55. Araya M, Figueira G, Espinoza J, Zarur X, Brunser 0 Acute diarrhoea and asymptomatic infection in Chilean preschoolers of low and high socioeconomic strata. Acta Paediatr Scand. 1986; 75(4): 645-51.

56. Fernández H, Martin R. Campylobacter intestinal carriage among stray and pet dogs. Rev Saúde Públ (São Paulo). 1991; 25(6):473-5.

57. Fernández $\mathbf{H}$. Occurrence of thermotolerant species of Campylobacter in three groups of hens maintained under different environmental conditions. Rev Microbiol (São Paulo). 1993; 24: 265-9.

58. Fernández, H, Hitschfeld M. Occurrence of Campylobacter jejuni and Campylobacter coli and their biotypes in beef and dairy cattle from the South of Chile. Braz J Microbiol. 2009; 40: $450-4$

59. Notario R, Borda N, Gambandé T, Sutich E. Species and serovars of enteropathogenic agents associated with acute diarrheal disese in Rosario, Argentina. Rev Inst Med Trop São Paulo. 1996: 8 (1): 5-7,

60. Giugno S, Oderiz S. Etiología bacteriana de la diarrea aguda en pacientes pediátricos. Acta Bioquím Clín Latinoam. 2010; 44 (1): 63-9.

61. López H, Valentina L. Diarrea aguda por Campylobacter en La Paz. Rev Boliv Pediatr. 1988; 27(1): 263-70.

62. Fernández H, Toledo MRF, Fagundes Neto U, Trabulsi LR. Occurrence of Campylobacter jejuni in diarrhoeic and non diarrhoeic children in São Paulo, Brazil. Rev Inst Med Trop São Paulo. 1985; 27(2): 102-4.

63. da Silva Quetz J, Lima IF, Havt A, de Carvalho EB, Lima NL, Soares AM, Mota RM et al. Campylobacter jejuni and Campylobacter coli in children from communities in Northeastern Brazil: molecular detection and relation to nutritional status. Diagn Microbiol Infect Dis. 2010; 67 (3):220-7.

64. Fernández H. Thermophilic species of Campylobacter: II Clinical, epidemiological and pathogenical aspects. Rev Chil Tecnol Méd. 1985;8: 301-9.

65. Figueroa G, Araya M, Ibañez S, Clecrc N, Brunser O. Enteropathogens associated with acute diarrhea in hospitalized infants. J Pediatr Gastroenterol Nutr. 1986;5:226-31.

66. Ordóñez SDM. Etiology of bacterial enteritis in humans: frequency of campylobacteriosis. Rev Colomb Pediatr Pueric. 1985; 36(2): 78- 93.
67. Manrique F, Billon D, Bello S, Ospina M. Agentes causantes de diarrea en niños menores de cinco años en Tunja, Colomia. Rev Peru Med Exp Salud Publica. 2006; 8 (1): 88-97.

68. Guderian RH, GR Ordoñez, RR Bossano. Acute diarrhea associated with Campylobacter and other pathogens in Quito, Ecuador. Bol Of Sanit Panam. 1987; 102(4): 333-9.

69. Weiler N, Franco R, Álvarez M, Zárate N, Chamorro G. Aislamiento y caracterización bioquímica de cepas de Campylobacter spp. en diarreas agudas en Paraguay. $6^{\circ}$ Congreso Paraguayo de Infectología. 2007; 1-3 de noviembre. Asunción, Paraguay.

70. Perales $\mathbf{M}$,Camiña $\mathbf{M}$, Quiñones $\mathbf{C}$. Infección por Campylobacter y Shigella como causa de diarrea aguda acuosa en niños menores de dos años en el distrito de La Victoria, Lima - Perú. Rev Peru Med Exp Salud Publica. 2002;19(4):186-92.

71. Castillo M, Gómez F, Laos M, Salinas M. Campylobacter spp en pacientes con cuadro diarreico que acudieron a Hospitales de la ciudad de Ica, Perú. Marzo-mayo 1999. Rev Peru Med Exp Salud Publica. 2003;20(3):S6.

72. Mota MI, Gadea MP, González S, González G, Pardo L, Sirok A, et al. Bacterial pathogens associated with bloody diarrhea in Uruguayan children. Rev Arg Microbiol. 2010; 42: 114-117.

73. Urrestarazu MI, Liprandi F, Pérez de Suárez E, González R, Pérez-Schael I. Características etiológicas, clínicas y sociodemográficas de la diarrea aguda en Venezuela. Rev Panam Salud Publica. 1999; 6 (3): 149-56.

74. Díaz C, Vizcaya L, Velasco J. Evaluación de un medio y la técnica de filtración para el aislamiento de Campylobacter sp. en un grupo de riesgo. Rev Fac Farm. 2003; 45 (1): 13-8.

75. Piazza D, Lasta JA. Presence of zoonotic campylobacters in cattle and swine for consumption in Argentina. Vet Res Commun. 1986; 10(1): 303-8.

76. Giacoboni G, López C, Tellechea D, Agostini A. Campylobacter jejuni en una granja de pollos camperos. Analecta Veterinaria. 2002;22(2):42-47.

77. Fernandez H. Espécies termófilas de Campylobacter: Aspectos bacteriológicos, epidemiológicos e patogênicos. [Tesis de Maestría]. São Paulo: Mestrado em Microbiologia e Imunologia. Escola Paulista de Medicina; 1983.

78. Modolo JR, Biping W, Kirpal K. Biochemical characters of Campylobacter $\mathrm{sp}$ isolated from calves with and without diarrhea. Rev Microbiol. 1988;19(1):1-5.

79. Kuana SL, Santos A, Rodrigues B, Borsoi A, Moraes C, Salle $\mathrm{C}$ et al. Occurrence and Characterization of Campylobacter in the Brazilian Production and Processing of Broilers. Avian Diseases. 2008; 52(4): 680-4.

80. Almeida PF, Serrano AM. Occurrence of Campylobacter fetus subsp jejuni in chickens and pigs carcasses. Rev Microbiol. 1987; 18(3): 279-83.

81. Andrade MCR, Lopes DA, Gabeira S.O, Esteves,WTC, Vilardo MCB, Thomé JDS, et al. Presença de Campylobacter spp. em macacos-de-cheiro (Saimiri spp.) assintomáticos cativos e sua correlação com as condições de manejo. Arq Bras Med Vet Zootec. 2009; 61(4): 890-5.

82. Filgueiras ALL, Hofer E. Diversity of Campylobacter isolates from three actived sludge systems. Mem Inst Oswaldo Cruz. 1998;93(3):295-8. 
83. Fernández H. Thermotolerant Campylobacter species associated with human diarrhea in Latin America. J Braz Ass Adv Sci. 1002; 44(1):39-43.

84. Figueroa G, Troncoso M, López C, Rivas P, Toro M. Occurrence and enumeration of Campylobacter spp. during the processing of Chilean broilers. BMC Microbiology. 2009; 9:94.

85. Tresierra A, Espinoza F, Macedo J, Bendayan ME, Bernuy A. Distribución de biovares Campylobacter jejuni ssp. jejuni y Campylobacter coli en monos de lquitos (Perú). Arch Med Vet. 1997;29(1):141-3.

86. Tresierra A, Bendayan ME. Thermotolerant Campylobacter species isolated from psittaciformes in the Peruvian amazon region. Rev Inst Med Trop S. Paulo. 1998; 40 (4): 263-4.

87. Tresierra A, Espinoza $E$, Bendayán $M$, Donayre $M$, Fernández $\mathbf{H}$. La fauna silvestre de la Amazonía peruana, un potencial reservorio de Campylobacter jejuni subsp. jejuni y Campylobacter coli. Folia Amazónica. 2006; 15 (1-2):117-22.
88. Olaguibel OL. Monitoreo de la resistencia antimicrobiana de Campylobacter spp. en cuatro hospitales de la ciudad de La Paz - Bolivia. [Tesis de Licenciatura en Bioquímica]. La Paz: Facultad de Ciencias Farmacéuticas y Bioquímicas, Universidad Mayor de San Andrés; 2009.

89. Ministerio de Salud, Dirección General de Salud de las Personas, Perú. Estudio de etiología de la diarrea en las direcciones de salud Cajamarca, Lambayeque, Loreto y Lima este. Informe Técnico. Lima: MINSA; 2001. 21 pp.

Correspondencia: Heriberto Fernández.

Dirección: Instituto de Microbilogía Clínica, Edificio de Ciencias Biomédicas, $2 .^{\circ}$ piso. Universidad Austral de Chile, Campus Isla Teja, Valdivia, Chile.

Correo electrónico: hfernand@uach.cl

\section{Suscríbase en forma electrónica y gratuita a los contenidos de la Revista Peruana de Medicina Experimental y Salud Pública, ingrese a WWW.ins.gob.pe, seleccione el ícono de la revista y envíenos sus datos.}

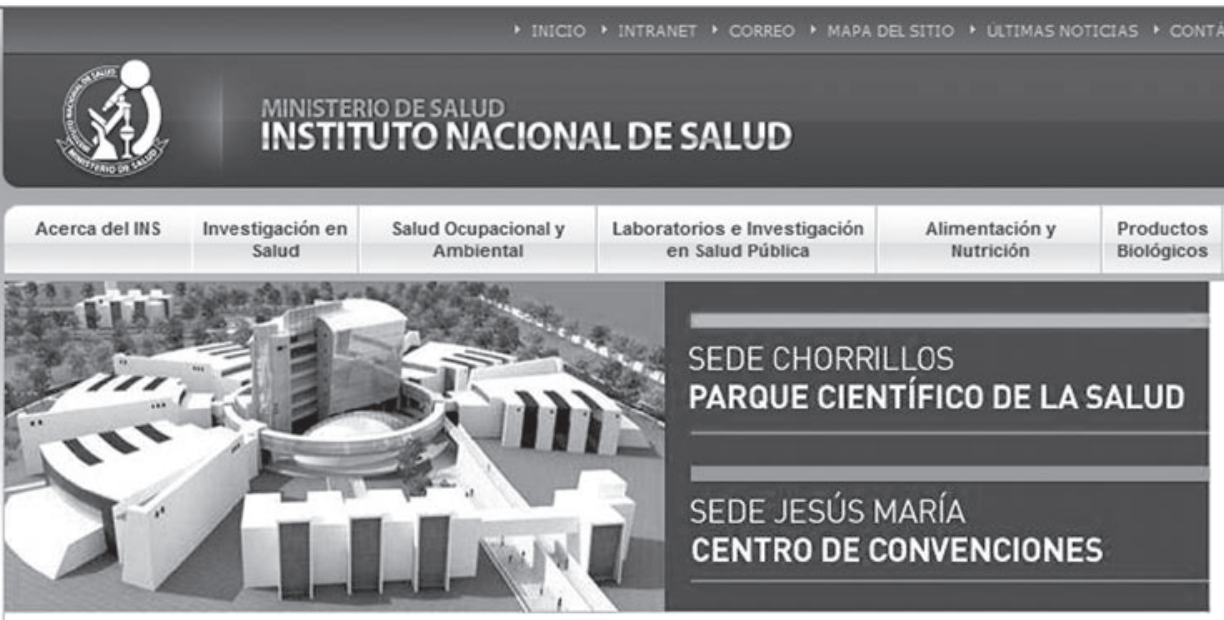

ÚLTIMAS INVESTIGACIONES Y EVIDENCIAS CIENTÍFICAS

$\underline{\text { Ver Todas }}$

Distribución de serotipos del virus dengue Perú 2011

La carga de Enfermedad y Lesiones en el Perú

Serotipos de dengue identificados por RT-PCR-TR en el Perú 2011 Ver detalle

Mortalidad, incidencias, prevalencias, duración de la enfermedad, discapacidad y años de vida saludables perdidos. Ver detalle

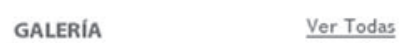

Imagen del día 2011

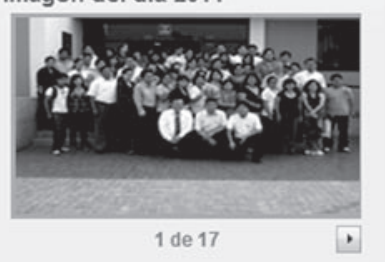
Intercultural de Medicamentos

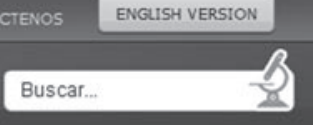

